\title{
Cloud characteristics and channel selection for IASI radiances in meteorologically sensitive areas
}

\author{
Nadia Fourrié and Florence Rabier \\ CNRM/GMAP METEO FRANCE, Toulouse, France
}

\section{Introduction}

Advanced infrared sounders, such as the Atmospheric InfraRed Sounder (AIRS, launched on board the Aqua satellite in May 2002) and the Infrared Atmospheric Sounding Interferometer (IASI) scheduled to be on board the first operational European polar-orbiting satellite Metop, measure or will measure radiation in many thousands of channels. They have the potential to provide atmospheric temperature and composition information at a much higher vertical resolution and accuracy that can be achieved with the previous generation of satellite instruments such as the High resolution Infrared Radiation Sounder. As it is neither feasible nor efficient to assimilate all the channels in a Numerical Weather Prediction (NWP) system, channel selection policies have been designed in the NWP context. Previous studies have proposed methods in order to reduce the number of channels to keep only the ones, which bring the most useful information. In particular Rabier et al. (2002) and Fourrié and Thépaut (2003) have used a method based on the information content following Rodgers (1996) for clear sky conditions.

If such advanced sounders have to lead to a very significant impact on the forecast quality, one should address in details the question of retaining the most information possible in the sensitive areas. Previous studies such as those of Prunet et al. (1998) and Collard (1998) have suggested that IASI could resolve some of the small scale baroclinic structures that have been identified by the sensitivity studies as being crucial to forecast error development (Rabier et al. 1996). Nevertheless, the main obstacle to use observation from advanced sounders is the presence of cloud, which can severely limit the information from infrared sounders. In this context, McNally (2002) previously investigated the occurrence of clouds in the sensitive areas with cloud fields from the European Centre for Medium-range Weather Forecasts model and showed with an "observable" sensitivity variable that there was a high correlation between the meteorologically sensitive areas and the cloud cover produced by the ECMWF model.

The first objective of this study is also to study the cloud cover and the cloud top level, but from the satellite imager AVHRR (Advanced Very High Resolution Radiometer) in simulated IASI pixels. This is done over the North Atlantic Ocean for cases of FASTEX (Front and Atlantic Storm-Track Experiment, Joly et al. 1999), and more particularly in the sensitive areas for the forecasts of storms of 8 Intensive Observing Periods (IOP). Once the cloud characteristics of the sensitive areas will be established, the robustness of the iterative channel selection method used by Rabier et al. (2002) (hereafter R02) and Fourrié and Thépaut (2003) will be addressed for these regions. This method will be compared with two channel selection methods using a criterion based on the adjoint sensitivity. These last two methods were developed in the context of the targeting problem, which consists in optimally selecting observations in the sensitive areas in order to reduce the error in the initial state of the forecast.

The next section presents the study of the cloudiness in the meteorological sensitive areas for the 8 FASTEX IOPs. The description of the channel selection framework and results obtained in a simple context of perfect observations for profiles with cloud top level at 1000 $\mathrm{hPa}$ from the FASTEX IOP sensitive areas are then discussed in section 3 . The channel 
selection methods are then applied in a more general context, always for these particular synoptic situations and a constant set is studied respectively in a new section. Finally, results are summarized and conclusions are given.

\section{Study of the cloudiness in the meteorological sensitive areas}

In this study, the cloud parameters (cloud cover and cloud top pressure in the simulated IASI pixels) have been retrieved from the AVHRR imager using the MAIA (Mask AVHRR for Inversion ATOVS) method, which was originally developed at the Centre de Météorologie Spatiale (CMS) in the frame of the AVHRR and ATOVS Processing Package (Lavanant et al, 1999).

The MAIA method has then been adapted to the IASI observations (Lavanant, personal communication). It processes the AVHRR observations mapped inside the simulated IASI pixel and determines the mean clear percentage cover in the IASI spot. With a succession of threshold tests applied to the AVHRR channels inside the simulated IASI pixel, it is possible to determine some cloud parameters. The cloud cover is evaluated from the percentage of clear AVHRR pixels in each simulated IASI ellipse shape. A minimum threshold of $90 \%$ of clear AVHRR spots has been arbitrarily chosen in order to consider this IASI pixel as clear. In addition, for the cloudy pixels, the cloud top temperature (if the cloud can be considered as a black body) among other parameters is available as outputs of the MAIA algorithm. The cloud top pressure level is deduced from the cloud top temperature with a series of differences between the cloud top temperature from the MAIA method and a temperature profile interpolated onto the simulated observation location. The temperature fields are available at every $50 \mathrm{hPa}$ in the vertical and are provided by the FASTEX 4D-Var reanalysis (Desroziers et al. 2003) including additional observations from the field experiment. The cloud top pressure level is found when the difference between the cloud top temperature and the atmospheric profile temperature is the smallest. It is worthwhile to note that only pixels over sea and for which a cloud top temperature could be retrieved have been considered in this study. Indeed, the MAIA method having been mainly tested over ocean owing to the CMS reception area, there is a lack of confidence in the method over iced land surfaces in the Halifax reception area.

\section{Determination of the sensitive areas}

The short-term forecast errors are mainly due to errors in the initial state of the forecast. In order to determine the regions where small errors in the initial state may increase and lead to major forecast errors, the gradient of a diagnostic function of the final state with respect to the initial conditions can be used (e.g. Rabier et al. 1996). Sensitive areas can be defined with scaled gradients resulting from these adjoint calculations. In this study, the gradient is based on the diagnostic function of the study from Hello et al.(2000). It is computed from the forecast of the mean sea level pressure over the area of the considered FASTEX weather systems. Eight dates on February 1997 have been studied. This period corresponds to a zonal weather regime, which is favourable to the development of cyclonic systems over western Europe (Joly et al. 1999 and Baehr et al. 1999). The temperature fields of the gradient at 900, 800, 700, 600 and $500 \mathrm{hPa}$ levels have been squared and summed at each geographical location in order to obtain a single horizontal sensitivity field. Once the sensitivity field has been computed, a threshold arbitrarily fixed at $30 \%$ of the sensitivity field maximum is applied to this field in order to obtain a horizontal mask for the observations located in the sensitive area for which the cloud cover and the cloud top height will be studied. It should be noted that the extent of the derived sensitive area slightly depends on this threshold. 


\section{Averaged results}

A brief description of the 8 FASTEX IOPs synoptic cases corresponding to some cases studied by Hello and Bouttier (2001) and Hello (2002) is given in Fourrié and Rabier (2002). The sensitive direction is computed with the forecast trajectory of the cyclones following the definition target and verification times, as described in Bergot (1999). The overall forecast range is mostly 36 hours and the verification area situated offshore of western Europe is centred on the location of the cyclones at the time of the verification. For six cases out of eight, the sensitivity maximum is located below or at the $700 \mathrm{hPa}$ level.

The IASI pixels have been simulated in the same area as the AVHHR observations, which are available from the Halifax reception station. These observations are representative of a mid-latitude region.

The averaged results of the distribution of the cloud top pressure for the sensitive areas and the whole dataset are presented in Fig. 1. For the sensitive areas, two maxima in the distribution of the cloud top level are identified for the atmospheric layers located between 1000 and $900 \mathrm{hPa}$ (low-level clouds) and 400-300 hPa (high-level clouds). Nonetheless, this synthetic table does not illustrate the case-to-case variability of the cloud top pressure in the sensitive area. In contrast, for the whole IASI dataset, almost $60 \%$ of the cloud tops of the observations are situated below $600 \mathrm{hPa}$. Furthermore, the cloud top pressure of observations of the mid-latitude area seems almost constant in time, mainly with low-level cloud. The sensitive areas are often located in the southern entrance area of an upper level jet-streak. Their horizontal extension depends also on the weather system. For almost all cases, the northern part of the sensitive area corresponds to low-level cloud and the southern one to high-level clouds. This particular shape of the cloud cover strongly limits an adequate sampling of the sensitive areas, the maximum of which is located at or below $700 \mathrm{hPa}$. These results corroborate the study of McNally (2002) who found a high degree of correlation between high cloud and sensitive areas during the winter.

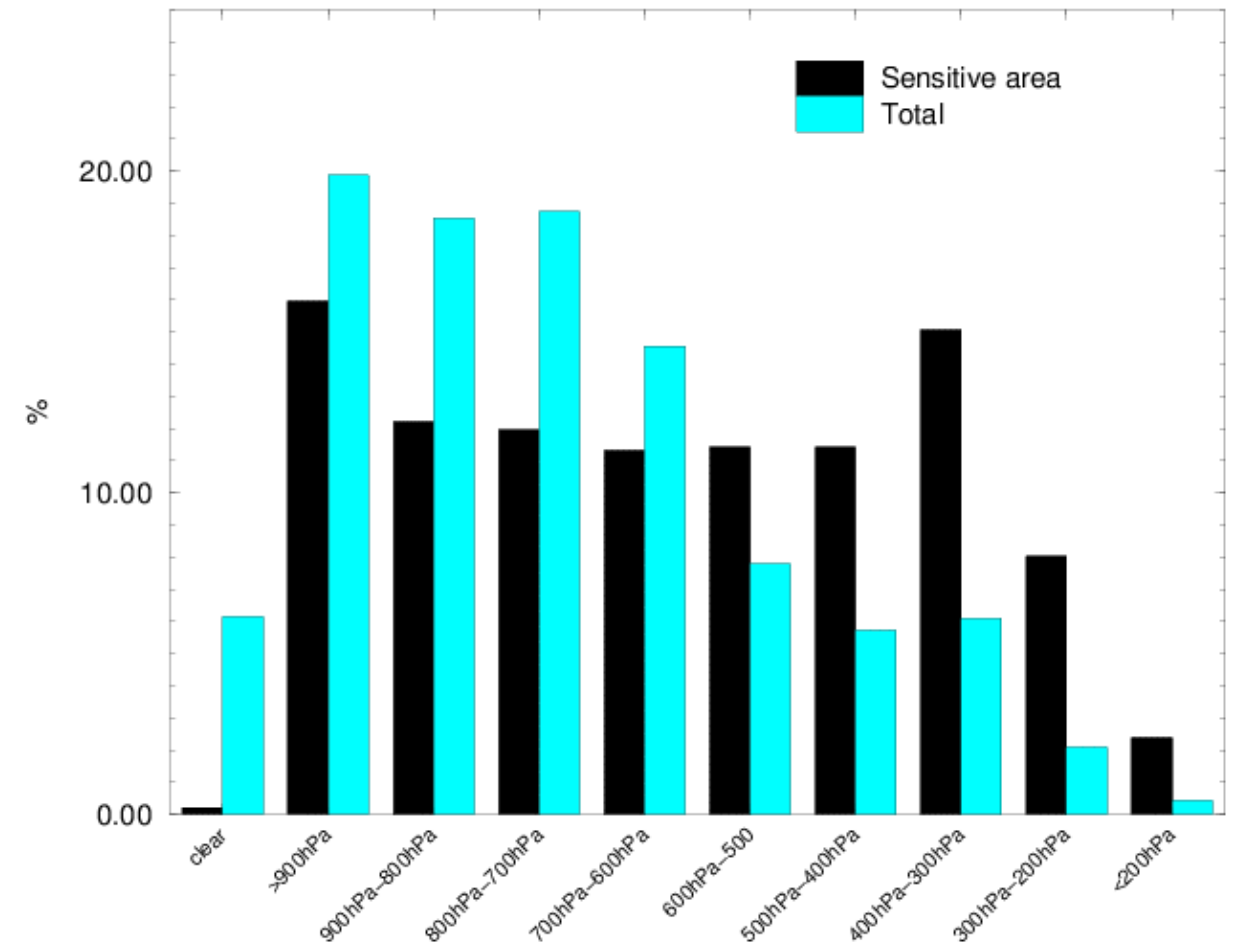

Fig. 1: Averaged distribution of the cloud top pressure as seen by the AVHRR imager in the IASI pixels for the sensitive areas (black bars) and for the whole set of pixels located in the mid-latitude area (blue bars). 
In conclusion, we have shown that a part of the simulated IASI pixels located in the sensitive areas were covered by low-level clouds and by high-level clouds. We will now more particularly tackle the question of the channel selection for these low-level cloud observation conditions (noting that the high-level cloud conditions are not likely to provide useful information from the infrared sounders).

\section{Description of the channel selections}

The general framework of this study is the linear optimal estimation theory in the context of NWP. The atmospheric profile in temperature, humidity and ozone at a given location is represented by a vector $\mathrm{x}$ and the satellite observations by a vector $\mathbf{y}$. The observations are linked to the atmospheric state by the radiative transfer equation:

$$
\mathbf{y}=\mathcal{H}(\mathrm{x})+\varepsilon_{\mathbf{O}}+\varepsilon_{\mathbf{F}}
$$

where the measurement and the forward model errors $\varepsilon_{\mathbf{O}}$ and $\varepsilon_{\mathbf{F}}$ are assumed to be gaussian noises with error covariance matrices $\mathbf{O}$ and $\mathbf{F}$. We will denote $\mathbf{R}=\mathbf{O}+\mathbf{F}$ the resulting observation error covariance matrix. The background state vector $\mathbf{x}_{\mathrm{b}}$ has an error covariance matrix denoted $\mathbf{B}$. The radiative transfer equation is assumed to be weakly non-linear, making the tangent linear assumption valid in the vicinity of the background state:

$$
\mathcal{H}(\mathbf{x})=\mathcal{H}\left(\mathbf{x}_{\mathrm{b}}\right)+\mathbf{H}\left(\mathbf{x}-\mathbf{x}_{\mathrm{b}}\right)
$$

where $\mathbf{H}$ is the tangent linear model of the radiative transfer model $\mathcal{H}$ (also called the jacobian matrix).

The optimal analysed state $\mathbf{x}_{\mathrm{a}}$ is given by $\mathbf{x}_{\mathrm{a}}=\mathbf{x}_{\mathrm{b}}+\mathbf{K}\left(\mathbf{y}-\mathbf{y}_{\mathrm{b}}\right)$ with $\mathbf{K}=\mathbf{A H R}^{-1}$ and

$$
\mathbf{A}=\mathbf{B}^{-1}+\mathbf{H} \mathbf{R}^{-1} \mathbf{H}^{-1}
$$

$\mathbf{K}$ is the Kalman gain matrix and $\mathbf{A}$ is the analysis error covariance matrix.

The parameter space is the temperature on the 43 pressure levels of the fast radiative-transfer model RTIASI (Matricardi and Saunders, 1999). The IASI data have been simulated from atmospheric profiles (temperature, humidity and surface temperature) for nadir views and noise has been added to the simulated data using the $\mathbf{O}$ covariance matrix provided by CNES in 2001. As in the R02 study, a constant error of 0.2K coming from the RT model noise is added to the measurement errors. The correlations existing between adjacent channels are ignored in the observation error matrix.

Only low-level clouds were tackled here. We have studied atmospheric profiles with a cloud top level at $1000 \mathrm{hPa}$ because the MAIA method found a maximum of low-level cloud top for this level. We assume cloud tops at the $1000 \mathrm{hPa}$ level with a surface emissivity of 1 . As our RT model is not assumed to be very accurate for the cloudy brightness temperature simulation and because there is an uncertainty on the cloud information coming from the accuracy of the MAIA algorithm, the channels sensitive to the cloud effects have been removed before the channel selection. The choice criterion is the jacobian: the test used for the determination of the cloud contamination is the ratio between the total jacobian surface and the jacobian surface below the cloud top level. If this ratio shows that more than $2 \%$ of the jacobian surface below cloud level, which corresponds to the 2 lowest levels of RTIASI for a cloud at $1000 \mathrm{hPa}$, the channel is considered to be too sensitive to the cloud to be kept in the channel selection. This allows the channels sensitive to the two last levels below $1000 \mathrm{hPa}$ to be removed from the channel selection and the number of channels is decreased from 8461 to 2300 channels. This number would be reduced to about 1600 channels if one considered cloud top level at $950 \mathrm{hPa}$. This prior channel selection is intended to keep the simulations as realistic as possible, assuming that the channel contaminated by cloud effects will be more difficult to use for the day-1 assimilation of IASI.

The problem of dynamical channel selection is tackled here. The iterative channel selection of R02 is compared to two methods developed in the framework of the targeting problem, which 
consists in determining where to select optimally some observations in order to reduce the forecast error variance.

\section{Entropy reduction}

This iterative method for channel selection, as proposed by Rodgers (1996) and used in R02, consists in performing successive analyses, each one using only channel at a time. The channel selection in our case is based on maximizing the Entropy Reduction (ER, Rodgers, 2000):

$$
E R=-1 / 2 \log _{2} \operatorname{det}\left(\mathbf{A B}^{-1}\right)
$$

The analysis error covariance matrix $\mathbf{A}$ is updated accordingly and is used at the next step as the background error covariance matrix. This ensures that all the information brought by previous channels is taken into account for the selection of the new channel. It will be called in the following the "ER method".

\section{Sensitivity to observations}

The sensitivity to observations (Baker and Daley, 2000 and Doerenbecher and Bergot, 2001) has been developed in the context of adaptive observation and these authors have suggested that the sensitivity with respect to observations could be an efficient tool for defining the location of targeted observations. An advantage of this kind of sensitivity is that it takes into account the way in which the observations will be assimilated through the multiplication of the gradient by the A matrix. Another advantage is that the sensitivity to observations is significantly reduced in regions already sampled by existing observations. This method will be applied to our problem of channel selection. Following the philosophy of the method, the channel $\mathrm{y}_{\mathrm{i}}$ selected is the one, which maximizes the sensitivity to observation:

$$
\nabla_{\mathbf{y o}} \mathrm{J}=\mathrm{ri}_{\mathbf{i}} \mathbf{h}_{\mathbf{i}}{ }^{\mathrm{T}} \mathbf{A}_{\mathbf{i}} \nabla_{\mathbf{x a}} \mathrm{J}
$$

where $\mathrm{r}_{\mathrm{i}}$ is the corresponding observation error value, $\mathbf{h}_{\mathrm{i}}{ }_{\mathrm{i}}$ is the vector corresponding to a line of the Jacobian matrix, $\nabla_{\mathbf{x}} \mathbf{J}$ is the sensitivity to the initial conditions and $\mathbf{A}_{\mathrm{i}}$ is computed from Eq. 3 for the corresponding channel $\mathbf{y}_{\mathbf{i}}$. As previously, this matrix is used at the next step as the background matrix in order to take into account the information brought by the already selected channels.

\section{Kalman Filter Sensitivity}

This Kalman Filter Sensitivity method was proposed by Bergot and Doerenbecher (2002) in order to find the optimal deployment of targeted observations. This method is based on the maximum decrease of the variance of the error on a given scalar function of forecast. The reduction in the variance of the error on a given scalar function of the forecast, due to the inclusion of one channel $\mathrm{y}_{\mathrm{i}}$ is given by:

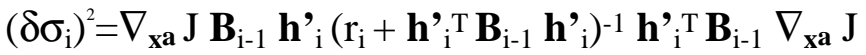

where $\mathbf{B}_{\mathrm{i}-1}$ corresponds to the analysis error covariance matrix obtained at the previous step. As for the ER method, the $\mathbf{A}$ matrix is updated and is used as the $\mathbf{B}$ matrix for the next step. This method is the so-called "KFS method".

\section{Key analysis errors matrix}

One month of "Key Analysis Errors" (KAE, as described in Klinker et al. 1998) has been computed at a resolution of T159 $(120 \mathrm{~km})$ : these "errors" represent perturbations that, if added to the ECMWF operational analysis, reduce the 48-hour forecast error (defined as the global difference between the 48-hour forecast and the verifying analysis). Up to now, humidity perturbations are not considered in the sensitivity computations, therefore only temperature is included in the sensitivity study described here. These structures generally are of small amplitude (meaning that a small atmospheric perturbation in this area can have a very large impact on the forecast quality) and can be fairly sharp both in the horizontal and in 
the vertical. The associated covariance matrix (averaged over one month) is sharper in the vertical and horizontal than the operational background covariance error. In addition, the error standard deviations are proportionally large in the troposphere in comparison with the ones of the stratosphere: the error maximum in the troposphere at about $300 \mathrm{hPa}$ is of the same order than in the stratosphere, when the error maximum in the troposphere for the "climatological" $\mathrm{B}$ matrix is two times smaller than the stratospheric maximum. This KAE covariance matrix has been chosen as the B matrix because our study is focussed on the channel selection in the sensitive areas.

\section{Results obtained with perfect observations}

In this first study, the truth is a "corrected state" which is a 3D-Var analysis modified with a scaled gradient perturbation. The coefficient applied to the perturbation was found by Hello (2002) in order to guarantee that inside the sensitive area the distance between the corrected state and the observations is minimal (Hello et al. 2000). Different observation sets can be used and the coefficient for which the forecast score based on verifying observations from the corrected state is the best, is chosen. The IASI measurements are simulated from this "corrected state". In order to better understand the manner in which the 3 channel selection methods work, no noise has been added to the observations. The background profile comes from the 3D-Var analysis ("uncorrected" by the gradient) and one tries to retrieve the sensitive perturbation with perfect observations of the reality. The $\mathbf{B}$ matrix used in the computation comes from the KAE matrix. We assume that KAE represent well perturbations from the gradient perturbations. As in the R02 study, 300 channels have been selected.

In order to study more precisely the channels selected by the three methods, attention has been paid to a single profile with a cloud top level of $1000 \mathrm{hPa}$ and close to the centre of the sensitive area. The results in term of background and analysis errors are shown in Fig. 2. The background error represents the difference between the original 3D-Var and the "corrected" analysis. It is larger in the $400-900 \mathrm{hPa}$ atmospheric layer and its maximum is located at about $550 \mathrm{hPa}$. Another relative maximum is present at $700 \mathrm{hPa}$. The three methods largely decrease the analysis error in comparison to the background one, although they slightly degrade the analysis in the 650-750 $\mathrm{hPa}$ atmospheric layer. In addition, the analysis error is slightly smaller for the two methods based on the adjoint gradient than for the ER method around the mean error peak while the ER method gives better results near the surface and in the high troposphere.

\section{Impact of a constant channel set}

Here, one wants to study the impact of a non-optimal channel set. As in R02, a constant channel set has been built from the ER method. The "constant" selection is computed as an average selection based on a set of representative mid-latitude situations. This set is part of the ECMWF atmospheric database (Chevallier et al. 2000) and forms a set of 170 vertical profiles of temperature, humidity, ozone together with values of surface pressure and surface temperature. The KAE matrix has been used as the $\mathbf{B}$ matrix during the channel selection process and a noise derived from the KAE matrix has been added in order to obtain the background state. This channel set has been used for the analysis of the 142 profiles located in the sensitive areas.

Before using the channels in the assimilation, it has been checked that these channels were not contaminated by clouds and the number of channels of the constant set actually used is close to 220 but depends on the profile. Figure 3 shows the results obtained with these two constant sets for the KAE matrix. A slight degradation is obtained in the troposphere in comparison with an optimal channel set. Moreover, the constant sets provide larger DFS or ER than the KFS set and a smaller rms ratio than the KFS set. These results suggest that an optimal channel set is not necessarily required for the analysis and that a constant set is a good compromise between the CPU cost and the quality of the analysis. 


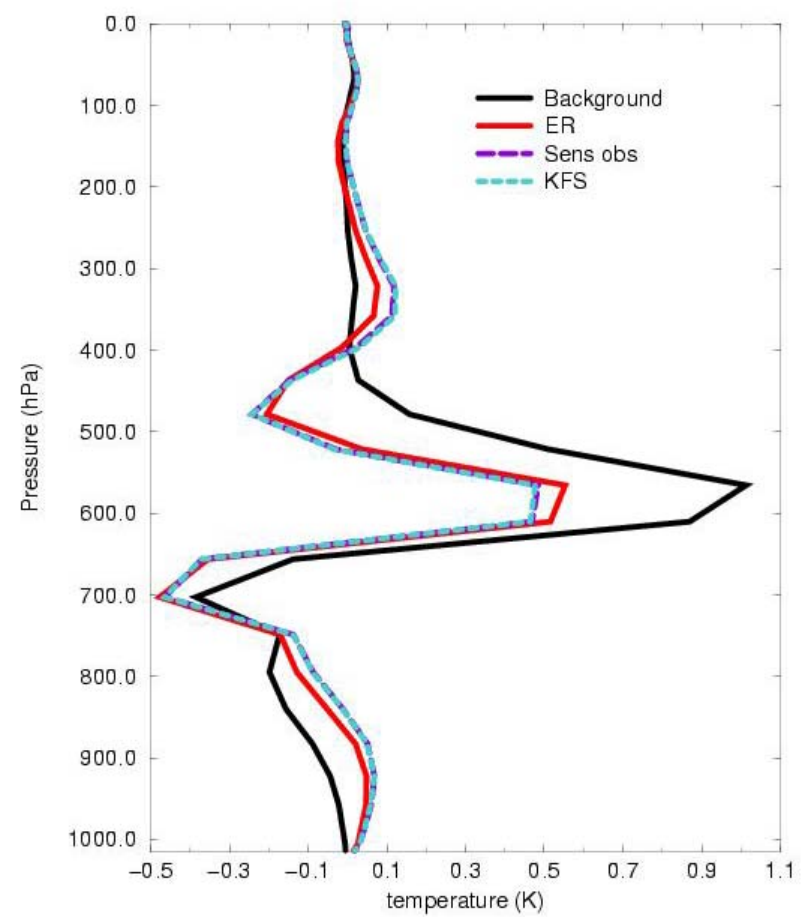

Fig. 2: Deviations from the true temperature for one particular representative profile for the background and the 3 different analyses. "Background" refers to the background error, "ER" to the analysis error obtained with the Entropy Reduction method, "Sens obs" to the analysis error given by the sensitivity to observation method and "KFS" to the analysis error produced by the Kalman filter Sensitivity method.

\section{Conclusions}

In this paper, the issue of reducing the number of IASI channels to be used in data assimilation has been specifically addressed in the context of the sensitive areas and for lowlevel clouds. In a first section, the cloud top level in simulated IASI pixels for FASTEX sensitive areas has been studied from the MAIA method using the AVHRR imager observations. It is found that the sensitive areas are usually covered by low-level clouds in their northern parts and by high-level clouds in their southern ones. These results corroborate the study of McNally (2002) who established a high correlation between the meteorologically sensitive areas and the cloud cover produced by the ECMWF model.

As a result of this first study, the channel selection has then been tackled in the context of low-level clouds $(1000 \mathrm{hPa})$ for the 8 FASTEX IOPs in order to retrieve information from the sensitive areas. The so-called iterative method of R02, based on the ER, has been compared to two methods derived from the targeting strategy that consists in adding optimal observations in the sensitive areas in order to reduce the error in the analysis, which can lead to a large forecast error. These methods are the sensitivity to observations and the Kalman Filter Sensitivity. In a first step, these three methods have been compared for the retrieval of one sensitive perturbation with perfect observations and it is shown that they provide similar results even though the ER selection and the other ones share few channels. 


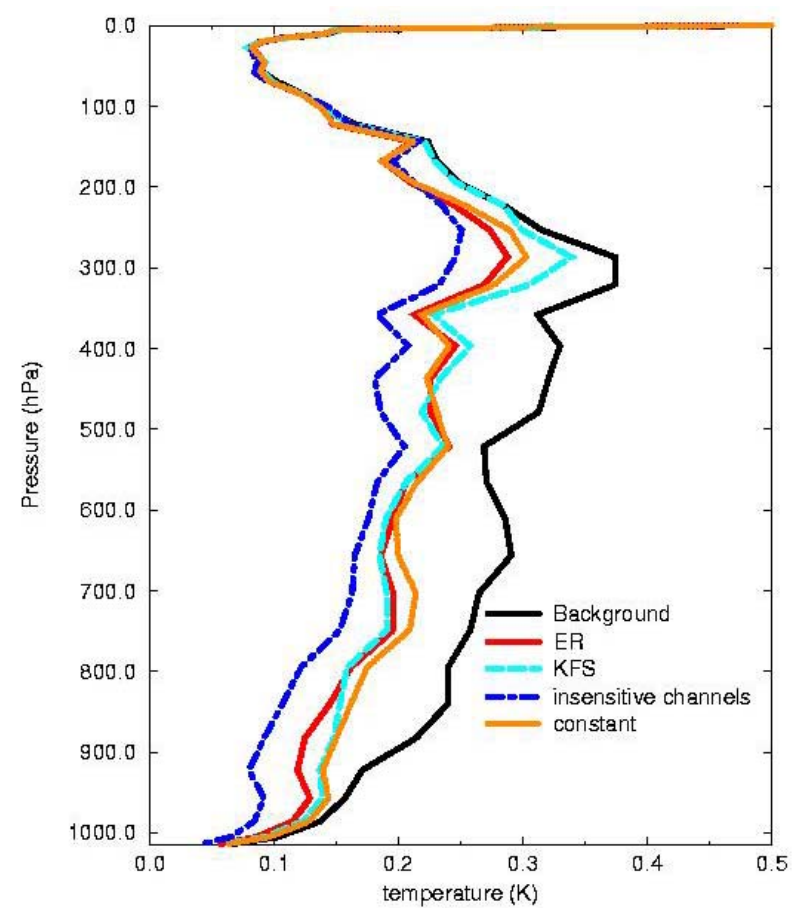

Fig. 3: Root mean square errors for 4 channel selection methods averaged over 142 profiles corresponding to a cloud top level of $1000 \mathrm{hPa}$. "Insensitive channels" corresponds to the assimilation of all the channels not contaminated by low-level clouds. "Constant" corresponds to an analysis computed with a constant channel set for clear sky conditions but the channels contaminated by cloud effect have been removed (about 220 channels depending on each profile)

As the sensitivity to observation channel selection method is very expensive in term of CPU cost, only the ER and KFS methods have then been compared from a statistical point of view over profiles from the FASTEX IOPs with low-level clouds. The ER method seems robust, even if the profiles are located within the sensitive areas. The use of a constant channel set computed from an independent set of atmospheric profiles still leads to a significant improvement of the analysis compared to the background even if this analysis error is slightly larger than the one obtained with an optimal channel set. More details about this study can be found in Fourrié and Rabier (2004).

Our main conclusions are: the ER channel selection method is quite robust, even for the studied highly sensitive profiles. In addition, the constant channel set deduced from this optimal channel selection can be computed from an independent atmospheric database, if it is representative of the profile air-mass. The ER channel selection method is very promising as previous results had not been focussed on such a characteristic set of profiles, especially crucial for the NWP. However, the first part of this study suggests that clouds are an important issue and that a radiative transfer model taking into account the cloud effect is required to go further in the channel selection studies in this context. One limitation of our work is that it took place out of the context of the operational NWP assimilation scheme and that the results of cloud level study in the sensitive areas are only valid for the limited number of the 8 FASTEX IOP synoptic situations and may have no general applicability to other situations and seasons. A future work could be the assimilation of the IASI radiances in a NWP assimilation scheme. This will provide the opportunity to test the real impact of the different channel selections on the forecast score, for which the gradient based channel selections are optimised. 


\section{Acknowledgment}

The study was partly supported by CNES trough post-doctorate fellowship of Nadia Fourrié. The authors wish to thank Gwenaëlle Hello (CNRM) who ran the computation of the gradient for the determination of the sensitive area. Tiphaine Labrot and Lydie Lavanant (CMS) are also acknowledged for having provided the cloud determination with the AVHRR observations interpolated onto IASI pixels for the Halifax area.

\section{References}

Baehr C., Pouponneau, B., Ayrault, F. and Joly, A. 1999. Dynamical characterization of the FASTEX cyclogenesis cases. Quart. J. R. Met. Soc., 125, 3469-3494.

Baker N. L. and Daley, R. 2000. Observation and background adjoint sensitivity in the adaptive observation targeting problem. Quart. J. R. Met. Soc.,126, 1431-1454.

Bergot T.1999. Adaptive observations during FASTEX: A systematic survey of upstream flights. Quart. J. R. Met. Soc., 125, 3271-3298.

Bergot T. and Doerenbecher, A. 2002. Study on the optimisation of the deployment of targeted observations using adjoint-based methods. Quart. J. R. Met. Soc , 128, 1689-1712.

Cammas J.-P., Pouponneau, B., Desroziers, G., Santurette, P., Joly, A., Arbogast, P., Mallet I., Cagnaux, G. and Mascart, P. 1999. FASTEX IOP 17 cyclone: Introductory synoptic study with field data. Quart. J. R. Met. Soc. , 125, 3393-3314.

Chevallier F. , Chédin A., Chéruy F. and Morcrette J.-J. 2000. TIGR-like atmospheric profile databases for accurate radiative flux computation. Quart. J. R. Met. Soc. ,126, 777-785.

Collard A. D., 1998. Notes on IASI performance, NWP Technical Report No. 253, Met Office, Bracknell, UK.

Desroziers, G., Hello, G. and Thépaut J.-N., 2003. A 4D-VAR Re-analysis of the FASTEX experiment, Quart. J. R. Met. Soc., 129. 1301-1315.

Doerenbecher, A. and Bergot, T. 2001. Sensitivity to observations applied to FASTEX cases. Nonlinear Processes in Geophysics, 8(6), 467-481.

Fourrié N. and Rabier F. 2002. Use of advanced infrared sounders in cloudy conditions. Proceedings of the Twelfth International TOVS Study Conference.

Fourrié N. and Rabier F. 2004. Cloud characteristics and channel selection for IASI radiances in meteorologically sensitive areas. Quart. J. R. Met. Soc., in revision, 18 p.

Fourrié, N. and Thépaut, J.-N., 2003. Evaluation of the AIRS Near Real Time channel selection for application to numerical weather prediction. Quart. J. R. Met. Soc., 128, 24252439.

Hello G., Lalaurette F. and Thépaut J.-N. 2000. Combined use of sensitivity information and observations to improve meteorological forecasts: A feasibility study applied to the 'Christmas Storm’ case. , Quart. J. R. Met. Soc., 126, 621-647. 
Hello, G., and Bouttier, F. 2001. Using adjoint sensitivity as a local structure function in variational assimilation. Nonlinear Processes in Geophysics. 8(6), 347-355.

Hello, G. 2002. Prise en compte de la dynamique associée aux dépressions des latitudes moyenne dans la détermination des conditions initiales des modèles météorologiques. $\mathrm{PhD}$ Thesis, Université Paul Sabatier, Toulouse III. 231 pp.

Joly, A. Browning K. A., Bessemoulin P., Cammas J.-P., Caniaux G., Chalon J.-P., Clough S. A., Dirks R., Emanuel K. A., Eymard L., Gall R., Hewson T. D., Hildebrand P. H., Jorgensen D., Lalaurette F., Langland R. H., Lemaitre Y., Mascart P., Moore J. A., Persson P. O., Roux F., Shapiro M. A., Snyder C., Toth Z. and Wakimoto R. M. 1999. Overview of the field phase of the Fronts and Atlantic Storm-Track EXperiment (FASTEX) project. Quart. J. R. Met. Soc. 125 877-946.

Klinker, E., Rabier, F. and Gelaro, R. 1998. Estimation of the key-analysis errors using the adjoint technique. Quart. J. R. Met. Soc. 124, 1909-1933.

Lavanant, L., Legléau, H., Derrien, M., Levasseur S., Monnier G., Ardouin L., Brunel P. and Bellec B. 1999. AVHRR Cloud Mask for Sounding Applications, Proceedings of the Tenth International TOVS Study Conference.

Matricardi M. and Saunders, R., 1999. A fast radiative transfer model for Infrared Atmospheric Sounding Interferometer radiances. Journal of Applied Optics. 38, 5679-5691.

McNally, 2002. A note on the occurrence of cloud in meteorologically sensitive areas and the implications for advanced infrared sounders. Quart. J. R. Met. Soc. 128, 2551-2556.

Prunet P., Thépaut J.-N. and Cassé V. 1998. The information content of clear-sky IASI radiances and their potential for numerical weather prediction., Quart. J. R. Met. Soc. 124, 211-241.

Rabier, F., Klinker, E., Courtier, P. and Hollingsworth, A. 1996. Sensitivity of forecast errors to initial conditions. Quart. J. R. Met. Soc. 122, 121-150.

Rabier, F., Fourrié, N., Chafaï D. and P. Prunet 2002. Channel selection methods for infrared atmospheric sounding interferometer radiances. Quart. J. R. Met. Soc. 128 1011-1027.

Rodgers, C. D. 1996. Information content and optimisation of high spectral resolution measurements. Optical Spectroscopic Techniques and Instrumentation for Atmospheric and Space Research II, SPIE Volume 2830, 136-147.

Rodgers, C. D. 2000. Inverse methods for atmospheres: Theories and practice. World Scientific Publ., Singapore, 238pp. 